\title{
Optimizing Process Parameters of Fused Deposition Modeling by Taguchi Method for the Fabrication of Lattice Structures
}

\author{
Guoying Dong, Grace Wijaya, Yunlong Tang, Yaoyao Fiona Zhao ${ }^{1}$ \\ McGill University, Department of Mechanical Engineering \\ 817 Sherbrooke Street West, Montreal, Quebec, H3A 0C3, Canada
}

\begin{abstract}
:
The lattice structure is a type of cellular material that can achieve a variety of promising physical properties. Additive Manufacturing (AM) has relieved the difficulty of fabricating lattice structures with complex geometries. However, the quality of the AM fabricated lattice structure still needs improvement. In this paper, the influence of parameters of the Fused Deposition Modeling (FDM) process on lattice structures was investigated by the Taguchi method. S/N ratio analysis was used to find the optimal process parameters that improve the printing quality, and ANOVA provided a significance ranking of the various factors analyzed in this paper. It was found that the optimum level and significance of each process parameter vary for horizontal and inclined struts. In addition, compression tests investigate the influence of process parameters on the mechanical properties of lattice structures. The results show that process parameters optimized by print quality can also improve the elastic modulus and the ultimate strength of these lattice structures.
\end{abstract}

Keywords: Additive Manufacturing, Fused Deposition Modeling, Lattice structure, Optimization, Taguchi method

\section{Introduction}

The lattice structure is a truss-like structure with intersecting struts and nodes with a certain repeated arrangement over a volumetric region. Compared to other cellular materials such as foams and honeycombs, lattice structures have more flexibility to achieve a variety of physical properties including high stiffness-weight ratio [1], low thermal expansion coefficient [2], negative Poisson ratio [3], and high heat dissipation rate through active cooling [4]. Due to these excellent characteristics, lattice structures have been extensively implemented in engineering applications, including ultralight structures [5, 6], energy absorbers [7], low thermal expansion structures [8], and conformal cooling [9]. In addition, lattice structures can be used as biocompatible materials for orthopaedic implant [10] and tissue engineering [11, 12].

In the past, the complexity of lattice structure design was severely restricted by traditional manufacturing techniques such as casting, sheet metal forming and wire bonding [13]. Only simple topologies and structures on a macro scale could be fabricated, unable to fully exploit the considerable potential of lattice structures. However, Additive Manufacturing (AM) technology provides an alternative approach to directly or indirectly [14] fabricate highly complex lattice structures via its layer by layer manufacturing principle. Powder bed fusion $[15,16]$ and material extrusion $[14,17]$ are the most common AM techniques for manufacturing lattice structures.

\footnotetext{
${ }^{1}$ Corresponding author, E-mail: yaoyao.zhao@ mail.mcgill.ca, Tel: +1 (514) 398-2523
} 
However, each manufacturing technology has its limitations, and AM is no exception. A wellknown limitation of AM is the need for support structures on down-facing surfaces [18]. Some support structures are difficult to remove, which leads to inferior surface quality or failed fabrication. To better understand the capability of AM to fabricate overhanging structures, the manufacturability and accuracy of self-supporting surfaces need further investigation.

A previous analysis on the effects of the process parameters of SLM on the roughness of selfsupporting surfaces [19] had limited experimental results and did not show a clear dependency on process parameters. Therefore, the relationship between process parameters of AM and the manufacturing quality of overhangs needs further analysis.

In addition, several attempts have been made to investigate process parameters of different AM approaches to enhance the print quality including surface roughness [20], density [21] and dimensional accuracy [22]. However, they focus on simple geometry rather than complex structures with many overhangs. Overhangs include horizontal struts and inclined struts, which are found in lattice structures and challenge the manufacturability of AM processes. Due to the lack of research relating the effect of process parameters on overhanging structures, there are no guidelines for finding the optimal process parameters of AM for the fabrication of lattice structures. Therefore, it is imperative to investigate the relationship between process parameters and the manufacturing quality of lattice structures to improve manufacturability.

In this paper, an optimization approach using the Taguchi method is proposed to find optimal parameters to fabricate lattice structures through Fused Deposition Modeling (FDM), a type of material extrusion AM process. The Taguchi method has been immensely used to optimize process parameters in product design through comprehensive experimental investigation. Since AM technology is influenced by many process parameters and currently has a high cost, the quantity and cost of a full factorial method experiment will be quite considerable. The design of experiment using Taguchi's method can significantly simplify the experimental plan. The Orthogonal Array (OA) is implemented here to give unique combinations between parameters and their levels to minimize the number of experiments while still investigating the entire parameter space.

In the Taguchi method, the analyses of experimental results have three primary objectives [23]. Firstly, studying the main effects of each factor gives the general influence of each factor. Secondly, by knowing whether higher or lower values produce the preferred result, the best levels of factors can be predicted. Finally, the contribution of each factor is established by analysis of variance (ANOVA), a statistical treatment to determine the relative percent influence and significance of each factor. After predicting the optimum conditions and expected performance, a confirmation test is usually conducted for verification. This is because the OA represents only a small fraction of all possibilities, so the optimum condition may not be present among the experimental combinations already carried out. Additionally, Signal-to-Noise $(\mathrm{S} / \mathrm{N})$ ratio analysis are conducted over the replicates to determine the most robust set of operating conditions from variations within the results.

In recent years, a lot of research have assessed the influence of the process parameters on the quality characteristics of FDM by the Taguchi method. For example, Anitha et al. [24] used the Taguchi method to investigate layer thickness, road width and the speed deposition to minimize the surface roughness. ANOVA revealed that layer thickness is the most significant parameter; and from S/N analysis, the optimum levels are $0.3556 \mathrm{~mm}$ layer thickness, $0.537 \mathrm{~mm}$ road width and $200 \mathrm{~mm}$ speed of deposition. Another study used the Taguchi method to evaluate the elastic performance and 
optimize the throwing distance of an FDM fabricated bow made with flexible ABS[25, 26]. It was found that air gap, slice height and raster angle most significantly impacted the elastic performance and optimum FDM parameter combinations were obtained. In addition, the Taguchi method with gray relational analysis has been used. One study used gray Taguchi to analyze the ultimate tensile strength, dimensional accuracy and surface roughness to optimize the FDM process [27]. The results demonstrate that for different objectives, the optimal combinations and most essential parameters may be different. Sood et al. [28] investigated the dimensional accuracy of FDM processed ABS400 part to minimize the percent change in length, width and height. The results indicated that optimum settings for each performance were different, so the gray relation grade combined three responses into a single response to simultaneously optimize parameters. The gray Taguchi method has also evaluated the influence of process parameters on the mechanical properties of FDM fabricated parts [29].

In this research, the design of experiment based on the Taguchi array evaluates the fabrication of lattice structures. $\mathrm{S} / \mathrm{N}$ ratio analysis finds the best level of process parameters, the ANOVA method investigates the significance of each process parameter, and the compression test evaluates the correlation between the print quality and mechanical performance of the lattice structure. This proposed approach is not limited to the FDM process; rather, the flow of experiments and method of data analysis is applicable to other AM processes for the selection of appropriate manufacturing parameters. This paper is organized as follows: Section 2 introduces the basic concepts used in this approach, Section 3 illustrates the design of experiments, and Section 4 presents the results of data analyses and compression test, and results discussion. Finally, the paper ends with conclusions and future research directions.

\section{Basic concepts}

\subsection{Manufacturable Element (ME)}

To link the design and manufacturing process of lattice structures, a concept called the Manufacturable Element (ME) [30] is used in this paper. This concept is defined by Rosen [31] as a predefined, parametrized decomposition of a volumetric region of a part. Applied to the characteristics of lattice structures focused in this paper, a ME of lattice structure is defined as a lattice strut with its related geometry, material and process information. To parametrically represent each ME of lattice structures, a data structure of ME is proposed and its graphic view is shown in Figure 1. The ME of lattice structures consists of geometrical data, material data and process data. The objective of this paper is to find the best process parameters for lattice structures fabricated by FDM. From the perspective of ME, it is to find the relationship between the process and geometrical data, while the material data is set to be constant. For better printing quality, the fabricated geometry should be as precise as the designed geometry. However, less error in the fabricated geometry does not mean a better mechanical performance. As a result, the compression test is also conducted to investigate the relationship between the mechanical properties of lattice structure and the process parameters. 


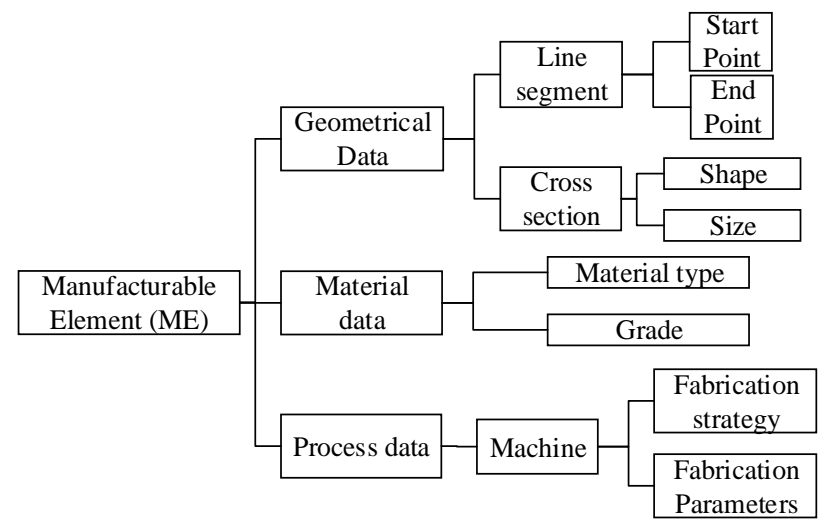

Figure 1. Graphic view of data structure of ME for lattice structures [30].

In this research, the samples are single struts, which is what lattice structures are constructed of. This is because it is difficult to evaluate the dimensional accuracy of the interior of as built lattice structures. Based on the geometrical data of ME, there are three types of lattice struts: horizontal struts, vertical struts and inclined struts. In addition, the cross section of the lattice strut will be circular, resulting in similar toolpaths for vertical struts and inclined struts [30]. Therefore, the vertical strut can be regarded as a special case of inclined struts with a 90 degree incline. Consequently, there are two lattice strut groups in this experiment: the horizontal group and the inclined group as shown in Figure 2. The controlled geometrical data include strut diameter for both groups, strut overhanging length for only the horizontal group, and strut incline angle for the inclined group.

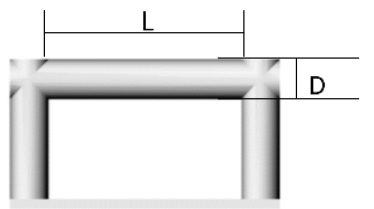

(a) Horizontal Group

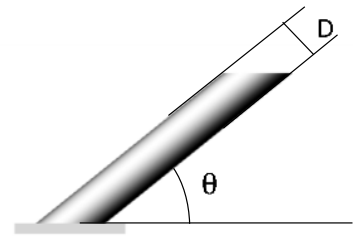

(b) Inclined Group

Figure 2. Examples of horizontal group and inclined group, $\mathrm{L}$ is the horizontal length, $\theta$ is the inclined angle, $\mathrm{D}$ is the diameter of both struts [30].

\subsection{Failure Method}

In the evaluation of the printing quality, $\Delta t$ is defined to represent the difference between the maximum and minimum thickness on a single strut. It is calculated by

$$
\Delta t=t_{\max }-t_{\min }
$$

The definition of $t_{\max }$ and $t_{\min }$ for both type of struts is shown in Figure 3. In general, the parts with the smallest difference had geometries closest to the modeled shape. However, because the levels tested a wide range of printing parameters, there were runs where the geometry was not printable or badly printed that cannot be measured as shown in Figure 4, resulting in missing information. Four different failure methods account for the missing data: omit the missing information, set $\Delta t$ to the maximum deviation of the parts of the same geometry, to the part diameter, and to twice the part diameter. These failure methods may overestimate or underestimate 
the quality of the failed strut. Careful comparison between these methods is necessary to find the optimal process parameters in the fabrication of lattice struts.

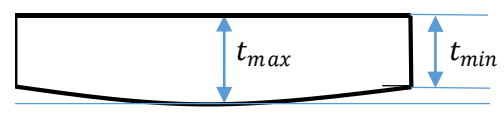

(a) Horizontal strut

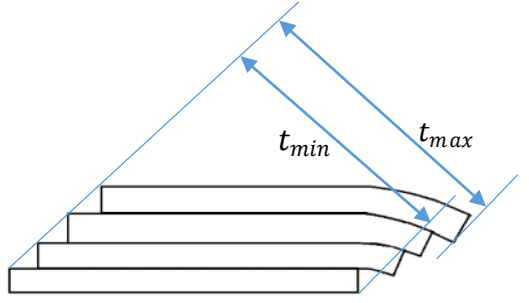

(b) Inclined strut

Figure 3. the difference between the minimum thickness $t_{\min }$ and the maximum fabricated thickness $t_{\max }$ for (a) horizontal strut and (b) inclined strut [30].

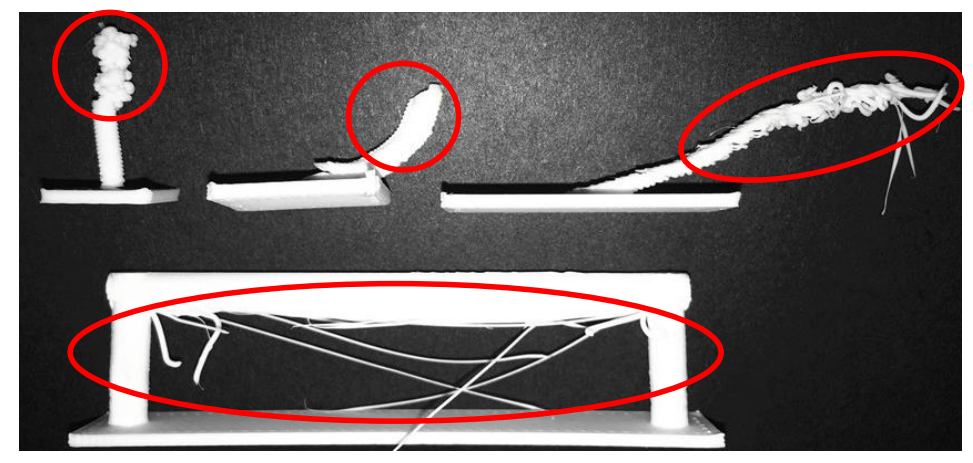

Figure 4. Poorly fabricated struts that are not measurable

\section{Design of Experiment}

In the experiment, the material data of ME is Acrylonitrile Butadiene Styrene (ABS) with no grade. Process data of ME include an Ultimaker 2 Extended+ for the machine, and FDM process for fabrication strategy. Based on the design parameters in Section 2.1, the lattice struts are divided into two groups: inclined group and horizontal group. Detailed information about the design parameters are summarized in Table 1. Before selecting the controlled process parameters, preliminary experiments are conducted to investigate the influence of process parameters on the manufacturing quality of lattice structures.

During preliminary experimentation, inclines and horizontal struts are printed under various process conditions to identify the most significant parameters. The parameters which created significant variations between parts are nozzle temperature, fan speed, print speed, and layer height. For each parameter, different levels are tested to find the range and levels to test. The range of the parameters are located at the limits of manufacturability, resulting in poor parts. In some cases, the machine's limitations define these limits - nozzle temperature hit a maximum at $255^{\circ} \mathrm{C}$, and fan speed can only be set between 0 and $100 \%$. The intervals are chosen to show a significant difference in part quality between levels. After preliminary testing, four printing parameters ( 2 with 4 levels, 1 with 3 levels, and 1 with 2 levels) were considered. Interactions between these parameters were 
not considered. The control parameters and their levels are summarized in Table 2. Other parameters held constant during the manufacturing process and are listed in Table 3. These parameters have little impact on part geometry during preliminary testing.

An orthogonal array is modified to test the design parameters. The initial array, L16, can hold a maximum of 215 or 45 factors. The linear graph in Figure 5 is used to merge nine 2 level columns into three 4 level columns [32]. This is done by combining the 2 level columns specified at the nodes of the linear graph, forming one 4 level column located in the column number specified by the line joining the nodes. To create the 3 level factor, dummy treatment changes 'level 4 ' to 'level $1 *$ ' [23]. Level 1 was chosen to be repeated for more runs because a fan speed of $0 \%$ is an absolute measure, whereas $50 \%$ and $100 \%$ are relative values that depend on the printer used. The orthogonal array is shown in

Table 4. Each set of parameters are used to print two types of structures: bridges with varying diameter and length, and inclined parts with varying diameter and angles listed in Table 1. Design parameters are tested with the full factor method. There are 9 horizontal samples and 12 inclined samples in each set of process parameters and each set is printed 3 times.

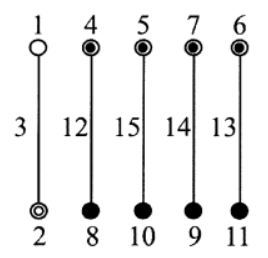

Figure 5. Linear graph used to merge nine 2 level columns, into three 4 level columns [32].

Table 1. Design parameters and their levels

\begin{tabular}{|l|l|l|l|l|l|l|}
\hline Geometry Group & Design Parameter & Units & Level 1 & Level 2 & Level 3 & Level 4 \\
\hline \multirow{2}{*}{ Inclines } & Angle from horizontal & $\circ$ & 15 & 30 & 60 & 90 \\
\cline { 2 - 8 } & Diameter & $\mathrm{mm}$ & 2 & 4 & 6 & - \\
\hline \multirow{2}{*}{ Horizontal Struts } & Length & $\mathrm{mm}$ & 10 & 30 & 50 & - \\
\cline { 2 - 7 } & Diameter & $\mathrm{mm}$ & 2 & 4 & 6 & - \\
\hline
\end{tabular}

Table 2. Controlled printing parameters and their levels

\begin{tabular}{|l|l|l|l|l|l|l|}
\hline Symbol & Printing Parameter & Unit & Level 1 & Level 2 & Level 3 & Level 4 \\
\hline A & Nozzle Temperature & ${ }^{\circ} \mathrm{C}$ & 225 & 235 & 245 & 255 \\
\hline B & Print Speed & $\mathrm{mm} / \mathrm{s}$ & 600 & 1200 & 1800 & 2400 \\
\hline $\mathrm{C}$ & Fan Speed & $\%$ & 0 & 50 & 100 & - \\
\hline $\mathrm{D}$ & Layer Height & $\mathrm{mm}$ & 0.1 & 0.2 & - & - \\
\hline
\end{tabular}

Table 3. Non-design process parameters

\begin{tabular}{|l|l|l|l|l|l|l|}
\hline Machine & $\begin{array}{l}\text { Bed } \\
\text { Temperature }\end{array}$ & $\begin{array}{l}\text { XY Travel } \\
\text { Speed }\end{array}$ & Z Speed & $\begin{array}{l}\text { Layer } \\
\text { Width }\end{array}$ & $\begin{array}{l}\text { Retraction } \\
\text { Distance }\end{array}$ & $\begin{array}{l}\text { Retraction } \\
\text { Speed }\end{array}$ \\
\hline $\begin{array}{l}\text { Ultimaker 2 } \\
\text { Extended+ }\end{array}$ & $100^{\circ} \mathrm{C}$ & $\begin{array}{l}3900 \\
\mathrm{~mm} / \mathrm{min}\end{array}$ & $\begin{array}{l}1000 \\
\mathrm{~mm} / \mathrm{min}\end{array}$ & $0.48 \mathrm{~mm}$ & $4.5 \mathrm{~mm}$ & $\begin{array}{l}1800 \\
\mathrm{~mm} / \mathrm{min}\end{array}$ \\
\hline
\end{tabular}


Table 4. Experimental runs with an L16 (42 x 31 x 21) orthogonal array

\begin{tabular}{|c|c|c|c|c|}
\hline Run & A & B & C & D \\
\hline $\mathbf{1}$ & 1 & 1 & 1 & 1 \\
\hline $\mathbf{2}$ & 1 & 2 & 2 & 2 \\
\hline $\mathbf{3}$ & 1 & 3 & 3 & 2 \\
\hline $\mathbf{4}$ & 1 & 4 & $1^{*}$ & 1 \\
\hline $\mathbf{5}$ & 2 & 1 & 3 & 1 \\
\hline $\mathbf{6}$ & 2 & 2 & $1^{*}$ & 2 \\
\hline $\mathbf{7}$ & 2 & 3 & 1 & 2 \\
\hline $\mathbf{8}$ & 2 & 4 & 2 & 1 \\
\hline $\mathbf{9}$ & 3 & 1 & $1^{*}$ & 2 \\
\hline $\mathbf{1 0}$ & 3 & 2 & 3 & 1 \\
\hline $\mathbf{1 1}$ & 3 & 3 & 2 & 1 \\
\hline $\mathbf{1 2}$ & 3 & 4 & 1 & 2 \\
\hline $\mathbf{1 3}$ & 4 & 1 & 2 & 2 \\
\hline $\mathbf{1 4}$ & 4 & 2 & 1 & 1 \\
\hline $\mathbf{1 5}$ & 4 & 3 & $1^{*}$ & 1 \\
\hline $\mathbf{1 6}$ & 4 & 4 & 3 & 2 \\
\hline
\end{tabular}

\section{Results and Discussion}

\subsection{Analysis of S/N Ratio}

$\mathrm{S} / \mathrm{N}$ ratios measure the variation in the data. Since the best case is to have no difference between the maximum and minimum dimensions of the data, the target $\Delta t$ value is 0 . Therefore, the $\mathrm{S} / \mathrm{N}$ ratio of $\Delta t$ is calculated by the lower-the better formula [23],

$$
\mathrm{S} / \mathrm{N}=-10 \log _{10}\left(\frac{1}{n} \sum_{i=1}^{n} \Delta t_{i}^{2}\right)
$$

The results are analyzed according to their geometry: inclines and horizontal struts. The process and design parameters varied for horizontal struts are temperature, print speed, fan speed, layer height, diameter, and length. The parameters varied for inclines are temperature, print speed, fan speed, layer height, angle, and diameter. The level response of each factor level is found by taking an average of the $\mathrm{S} / \mathrm{N}$ ratios within that factor level.

$$
A=\frac{1}{n_{k}} \sum_{i=1}^{n_{k}} S / N_{i}
$$

In addition, the response range for each factor was calculated by

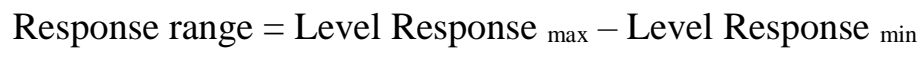

The main effects of different process parameters at different levels are shown in Figure 6 for inclined struts and Figure 7 for horizontal struts. A higher S/N ratio indicates better printing quality. The main effects charts demonstrate that the failure methods of taking maximum deviation, strut diameter, and twice the strut diameter follow similar trends; while the omitted method does not. This is because the failed parts have extremely poor printing quality which is not reflected when 
results are omitted. The omission will underestimate the negative influence of process parameters on those failed parts. Therefore, taking the $\Delta t$ equal to maximum deviation, strut diameter, and doubling the strut diameter are more reasonable approximations to treat missing data due to failed prints and will be considered to find the optimal process parameters. However, there are still some inconsistencies between these three failure methods for some parameters, so additional experiments are conducted to further investigate those parameters and will be discussed in the next subsection.
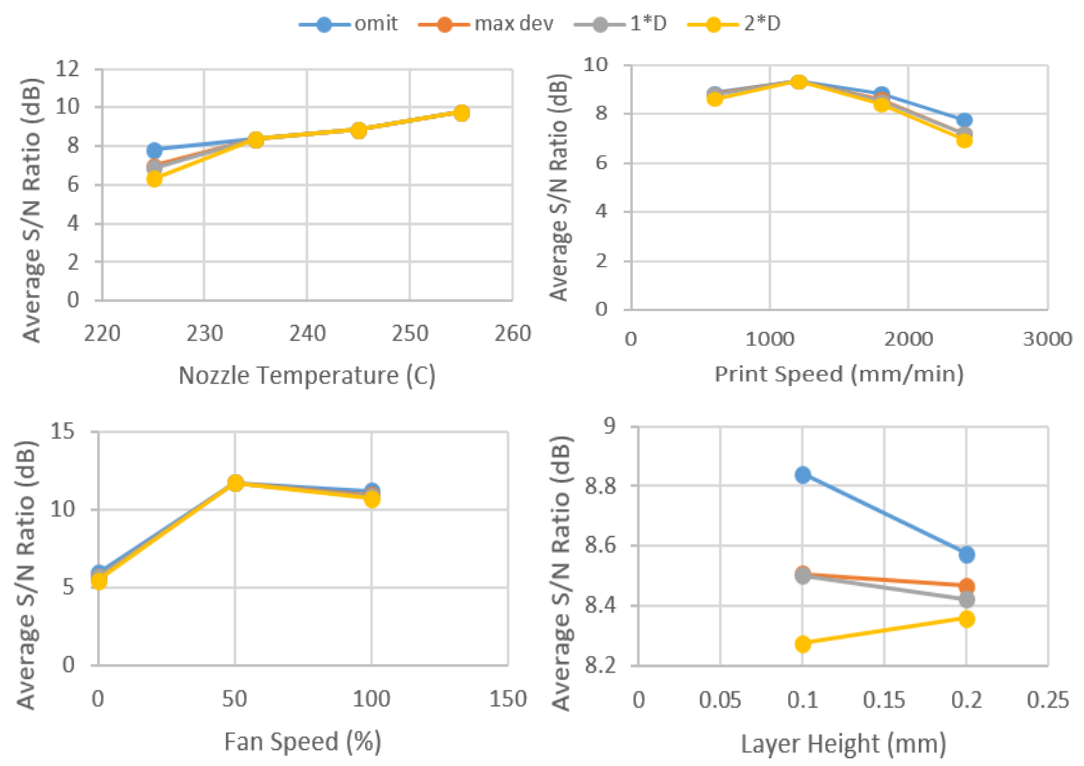

Figure 6. Main effects charts for the effect of process parameters on average S/N Ratio for inclines
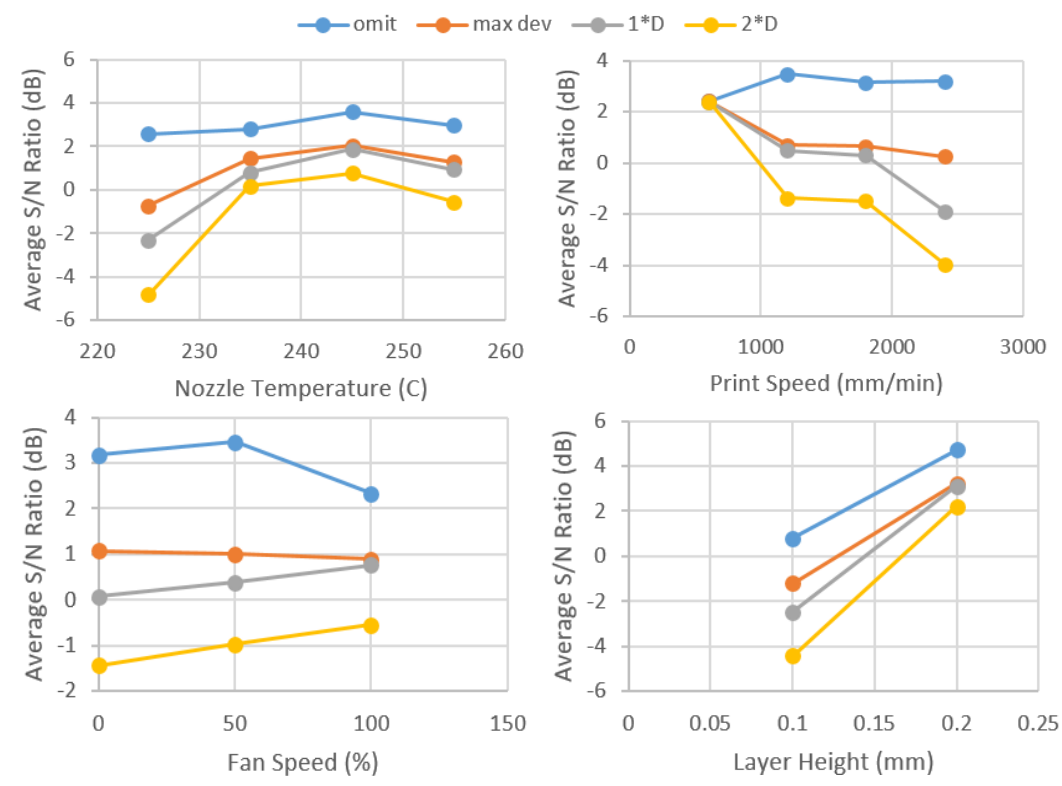

Figure 7. Main effects charts for the effect of process parameters on S/N Ratio for horizontal struts

The optimal levels of the process parameters can be selected from Figure 6 and Figure 7, indicated by the levels which produce the highest $\mathrm{S} / \mathrm{N}$ ratio values. For inclined struts, the trends for nozzle temperature, printing speed and fan speed are the same for all the failure methods. That means the optimal level for those parameters can be determined by the failure methods. The optimal levels 
are level 4 or $255^{\circ} \mathrm{C}$ for nozzle temperature, level 2 or $1200 \mathrm{~mm} / \mathrm{min}$ for print speed, and $50 \%$ for fan speed. The best level for layer height cannot be determined because the trends for the $\mathrm{S} / \mathrm{N}$ value are different in the three failure methods. This indicates that some failure methods may overestimate or underestimate the influence of the layer thickness on inclined struts.

The print failure of struts interferes with the determination of optimal process parameters. The failure is because the OA cannot guarantee combinations of good printing quality. However, since the other three optimal process parameters have been found, the three settings can be used to refabricate the inclined struts with better printing quality regardless of layer thickness. This will eliminate the influence of the failed samples, and the optimal level of layer thickness for inclined struts can be found. This additional experiment is conducted in the confirmation test to further investigate the undetermined process parameters.

As for horizontal struts, three parameters: nozzle temperature, layer height and printing speed can be determined by failure methods while the fan speed shows some inconsistency among failure methods. Through finding the highest $\mathrm{S} / \mathrm{N}$ ratio for those three process parameters in Figure 7, it is found that the best level for nozzle temperature is level 3 or $245^{\circ} \mathrm{C}$, for printing speed is level one or the slowest speed, and for layer height is level 2 or $0.2 \mathrm{~mm}$ printing thickness. However, for the fan speed, different failure methods show different trends, for the reasons explained above. Therefore, additional confirmation testing is required to determine the optimal fan speed for horizontal struts.

\subsection{Confirmation test}

In Section 4.1, the main effect charts did not show consistency for the layer thickness of inclined struts, and fan speed of horizontal struts between the three different failure methods. Additional tests are conducted to further investigate the optimal level of layer thickness for inclined struts and fan speed for horizontal struts. Five additional tests are designed and the process parameters of each test are shown in Table 5. For inclined struts, the layer height is set to $0.1 \mathrm{~mm}$ and $0.2 \mathrm{~mm}$, and for horizontal struts, the fan speed was set to $0 \%, 50 \%$ and $100 \%$. The other parameters were set at the optimal level for inclined and horizontal struts respectively as obtained in the S/N Ratio analysis. The optimal level chosen will minimize print failure in these five tests.

Table 5. Process parameters in additional tests

\begin{tabular}{|c|c|c|c|c|c|}
\hline & Test No. & nozzle temp & print speed & fan speed & layer height \\
\hline \multirow{3}{*}{ Inclines } & 1 & \multirow{2}{*}{255} & \multirow{2}{*}{1200} & 50 & 0.1 \\
\cline { 2 - 2 } & 2 & & & 0.2 \\
\hline \multirow{3}{*}{ Horizontals } & 3 & \multirow{2}{*}{245} & \multirow{2}{*}{600} & 0.2 \\
\cline { 2 - 2 } & 4 & & & \multicolumn{2}{|c}{50} \\
\cline { 2 - 2 } & 5 & & & 100 & \\
\hline
\end{tabular}

The results of these tests are shown in Figure 8. A higher $\mathrm{S} / \mathrm{N}$ ratio refers to a better print quality. It can be concluded that the $0.1 \mathrm{~mm}$ layer height is better than $0.2 \mathrm{~mm}$ layer height for inclined struts. This is because slicing over a smaller layer height will reduce the overhang length of the layer. Additionally, the $0 \%$ fan speed is better than $50 \%$ and $100 \%$ for horizontal struts. During the print, the first layer is very weak because of the large overhang, and the fan's airflow in the printing chamber will cause oscillation of the unsolidified layer. Therefore, reducing airflow without a fan 
will result in better printing quality for the horizontal struts. Via $\mathrm{S} / \mathrm{N}$ ratio analysis and confirmation test, the optimal level of each process parameter is obtained and summarized in Table 6 . It is found from the result that best process parameters for different types of the lattice struts are totally different, due to the differing geometrical characteristics between horizontal struts and inclined struts. Therefore, if the parameters are dynamic during the printing process to optimize for different types of lattice struts, the printing quality of the entire structure can be further improved. In addition, tests No.1 and No. 3 can be regarded as the confirmation test for inclined struts and horizontal struts because all the process parameters are optimum. It can also be concluded that the $\mathrm{S} / \mathrm{N}$ ratio of the optimized process parameter is larger than the S/N ratio of the L16 array, which means that the optimized result improves the printing quality of both horizontal and inclined struts.

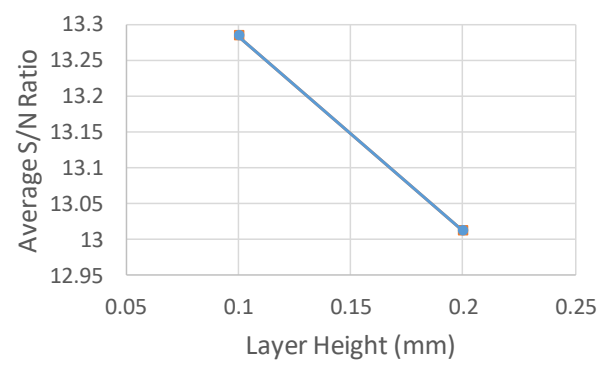

(a) inclined strut

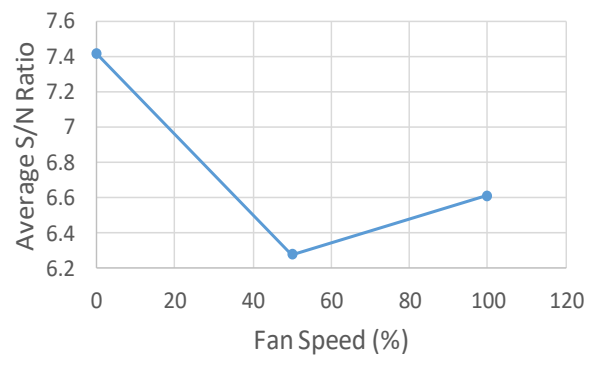

(b) horizontal strut

Figure 8 . The $\mathrm{S} / \mathrm{N}$ result of additional tests

Table 6. Optimal process parameters for inclined struts and horizontal struts

\begin{tabular}{|c|c|c|c|c|}
\hline Inclined struts & $\begin{array}{c}\text { nozzle } \\
\text { temp }\end{array}$ & $\begin{array}{c}\text { print } \\
\text { speed }\end{array}$ & $\begin{array}{c}\text { fan } \\
\text { speed }\end{array}$ & $\begin{array}{c}\text { layer } \\
\text { height }\end{array}$ \\
\hline Horizontal struts & 255 & 1200 & 50 & 0.1 \\
\hline
\end{tabular}

\subsection{ANOVA}

Analysis of variance for means is performed on the data to find the most significant process parameter for both inclined and horizontal struts. Three sets of ANOVA tables are calculated for each geometry type based on three failure methods which are maximum deviation, strut diameter, and doubling the strut diameter. Interactions are not considered as factors were assumed to be independent. Degrees of freedom for factor A is calculated by

$$
\mathrm{df}_{A}=\mathrm{m}-1
$$

Where $\mathrm{m}$ is the total levels of A. The total degrees of freedom is the number of observations, subtracted by 1 . The number of samples are 576 and 432, for inclines and horizontal struts respectively. Therefore, $\mathrm{dfTot}_{\text {inclines }}=575$, and $\mathrm{dfTot}_{\text {hor }}=431$. Finally, degrees of freedom for error is calculated by

$$
\mathrm{dfE}=\mathrm{dfTot}-\sum_{i=1}^{n} d f A_{i}
$$

where $\mathrm{n}$ denotes the total number of factors, and $\mathrm{df} \mathrm{A}_{\mathrm{i}}$ is the degree of freedom for each factor. The mean $\Delta t$ is calculated for each set of replicated trials, where the levels for each factor are held constant. This is calculated by 


$$
\overline{\Delta t}_{\mathrm{i} .}=\frac{1}{n} \sum_{j=1}^{n}\left(\Delta t_{i j}\right)
$$

where $\mathrm{n}$ is the number of observations values within the level. The grand mean is calculated by

$$
\overline{\Delta t}_{. .}=\frac{1}{n k} \sum_{i=1}^{n} \sum_{j=1}^{k}\left(\overline{\Delta t}_{i j}\right)
$$

where $\mathrm{i}$ and $\mathrm{j}$ represent all the factors and their levels respectively. The sum of squares of factor $\mathrm{A}$ is obtained by [33]

$$
\mathrm{SS}_{\mathrm{A}}=N \sum_{i=1}^{n}\left(\overline{\Delta t}_{i .}-\overline{\Delta t}_{. .}\right)^{2}
$$

In this case, $\mathrm{N}$ is the number of observations at each factor level. The fan speed parameter requires unbalanced ANOVA (Eq. 10) because the dummy variable used doubles the number of observations for a fan speed of $0 \%$.

$$
\mathrm{SS}_{\mathrm{A}}=\sum_{i=1}^{n} N i\left(\overline{\Delta t}_{i .}-\overline{\Delta t}_{. .}\right)^{2}
$$

where $N i$ is the number of observations of each factor level $\mathrm{i}$. Then the mean sum of squares can be obtained:

$$
M S_{A}=\frac{S S_{A}}{d f_{A}}
$$

The total sum of squares is calculated by Eq. 12 for each set of data: inclines and horizontal struts

$$
\mathrm{SS}_{\mathrm{T}}=\sum_{i=1}^{n} \sum_{j=1}^{k}\left(\Delta t_{i j}-\overline{\Delta t}_{. .}\right)^{2}
$$

The sum of squares error is given by

$$
\mathrm{SS}_{\mathrm{err}}=\mathrm{SS}_{\mathrm{T}}-\sum_{i=1}^{n} S S_{A, i}
$$

where $\mathrm{n}$ is the total number of factors and $M S_{A, i}$ is the mean sum of squares for each factor. The $\mathrm{F}$ ratio of factor is calculated by

$$
\text { F ratio } \text { A }=\frac{S S_{A}}{S S_{\text {err }}}
$$

P-contribution, is the mean sum of squares of a factor divided by the total mean sum of squares of all the factors, including error. It is calculated by Eq. 15.

$$
\mathrm{P}_{\mathrm{A}}=\frac{M S_{A}}{\sum_{i=1}^{n} M S_{A, i}}
$$

where $\mathrm{n}$ is the total number of factors, and $M S_{A, i}$ is the mean sum of squares for each factor. The results of the AONVA through three failure methods are shown in Figure 9 and Figure 10 for inclined struts and horizontal struts, respectively. The contributions of each factor for horizontal struts and inclined struts are different. Small error contributions indicate that it is reasonable to assume no interactions between factors. Although the P-value varies slightly for different failure methods, the process parameters which gives the maximum P-value do not change with failure method. Furthermore, the rank of the influence is almost the same, except ranks 3 and 4 in the maximum deviation method for horizontal struts. However, it can be concluded that these parameters have little influence on the printing quality of horizontal and vertical struts.

The average $\mathrm{P}$ values demonstrate that for inclines, the contribution of fan speed is $81.00 \%$, nozzle temperature is $11.97 \%$, print speed is $3.05 \%$, and layer height is $1.42 \%$. For horizontal struts, the contribution of layer height is $69.84 \%$, temperature is $15.14 \%$, print speed is $11.52 \%$, and fan speed is $1.74 \%$. Therefore, the most significant process parameters are fan speed for inclined struts and 
layer height for horizontal struts. The reason is that for inclined struts, the overhang area can be cooled down quickly by the fan which maintains the overhang's shape. Without a fan, the overhang area remains soft and will sag due to the gravity. As for horizontal struts, the first layer of the overhang is very important. With a larger printing thickness, the first layer of the overhang is more robust to support the following layers. Therefore, these two process parameters are significant for inclined struts and horizontal struts respectively.

Figure 11 summarizes average $\mathrm{S} / \mathrm{N}$ value ranges, in which a higher value indicates greater influence on the printing quality. By comparing ANOVA and $\mathrm{S} / \mathrm{N}$ response range, the ranks for the $\mathrm{S} / \mathrm{N}$ value range and $\mathrm{P}$ contribution are similar, and the largest range between the lowest and highest $\mathrm{S} / \mathrm{N}$ values in all levels of the factor correspond to the largest contribution of that factor. It can also be concluded that the most significant process parameter for both types of struts obtained by the S/N value range is the same as obtained by ANOVA analysis. Therefore, both $\mathrm{S} / \mathrm{N}$ value range and ANOVA analysis can be used to determine the significance of each parameter. The influence of these parameters on the mechanical property of lattice structures will be discussed in the next subsection.
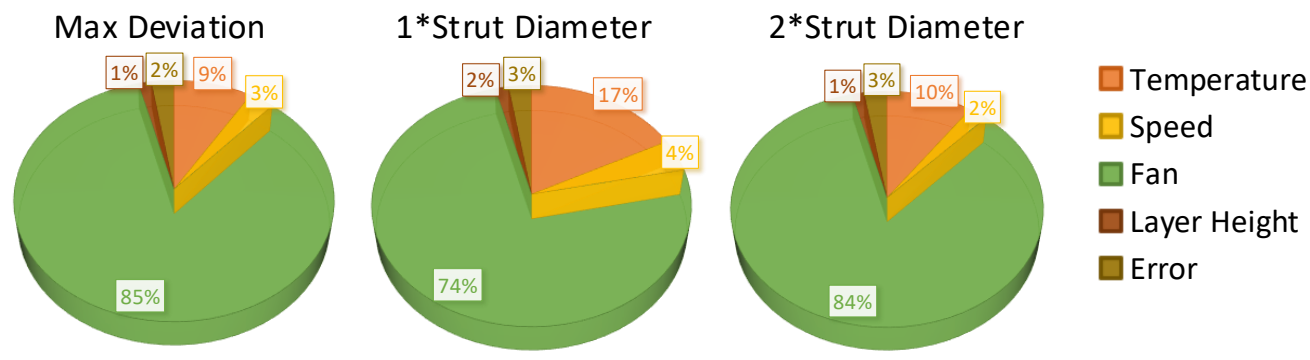

Figure $9 \mathrm{P}$ values for inclined struts for three failure methods respectively
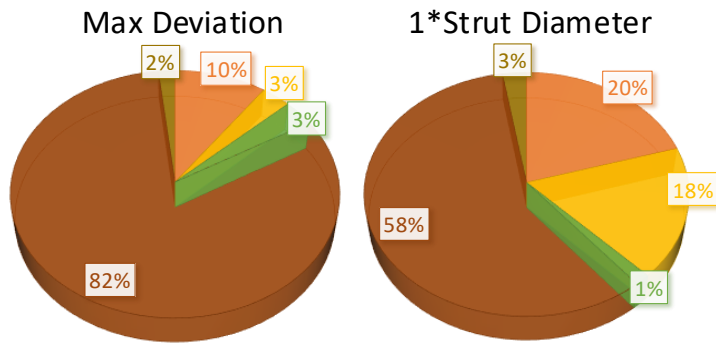

$2 *$ Strut Diameter

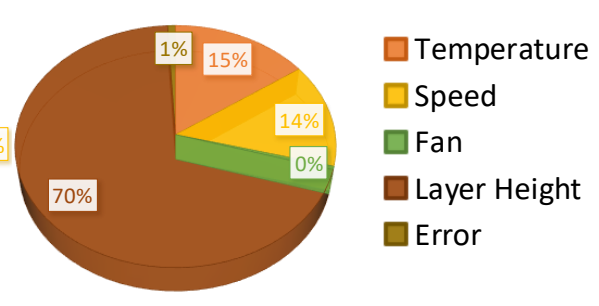

Figure $10 \mathrm{P}$ values for horizontal struts for three failure methods respectively 


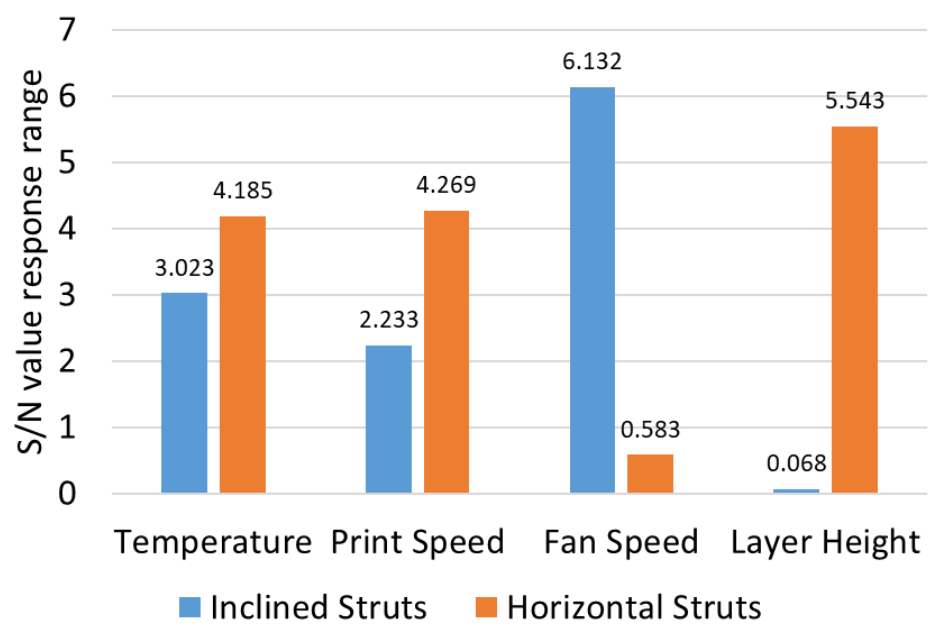

Figure 11 Average $\mathrm{S} / \mathrm{N}$ value response range for inclined and horizontal struts, a higher value means these parameters has more influence on the printing quality

\subsection{Mechanical Test}

\subsubsection{Fabrication of lattice structures}

After S/N ratio analysis and ANOVA, the optimal process parameters for both horizontal struts and inclined struts are obtained, and the most significant parameter for each type of strut is determined. However, these analyses only focus on single struts and dimensional accuracy. To understand the influence of the process parameters on the entire lattice structure, the compression test is conducted to evaluate the mechanical performance of lattice structure fabricated by different process parameters. This test also verifies whether process parameters optimized by dimensional accuracy improves mechanical performance. In this experiment, geometrical data is set to be constant. All the lattice structures have the same dimension with a cubic-center topology with horizontal, inclined, and vertical struts. The length of the unit cell is $15 \mathrm{~mm}$ and the strut thickness is $2 \mathrm{~mm}$. The lattice structure has three unit cells along each direction, as shown in Figure 12(a). The relative density is $12.8 \%$.

The lattice structure is fabricated with the process parameters in

Table 4, and each run is shown in Figure 12(b). Half the fabrications have failed due to the process parameters in OA. If the optimization process had been directly applied on the entire lattice structure instead of single lattice struts, half the data would not be obtainable for the S/N ratio analysis and ANOVA. This demonstrates the benefit of using single struts, as most struts are still measurable and the missing data is only a small portion of the whole. Via the failure methods proposed in this research, the S/N ratio and ANOVA methods can find the optimal process parameters and the most significant process parameters. In contrast, manufacturing error accumulates in an entire lattice structure at each layer, which leads to the failure of subsequent struts. Therefore, it is difficult to optimize the process parameters due to plenty of failed fabrications.

To verify the improvement of optimization process, the lattice structure is also fabricated by the optimized process parameters found from S/N ratio analysis and ANOVA. Because optimal levels of horizontal struts and inclined struts are different, overall optimal parameters have to be 
determined. From ANOVA, the most significant parameter is fan speed for inclined struts and layer thickness for horizontal struts. Therefore, the fan speed is set to $50 \%$, the best level for inclines; and the layer thickness set to is $0.2 \mathrm{~mm}$, the best level for horizontal struts. Printing speed has little influence on the inclined struts, so $600 \mathrm{~mm} / \mathrm{min}$ is selected because it is the best level for horizontal struts. The temperature has equivalent significance for both types of struts and an average value of the two optimal temperatures is chosen, which is $250^{\circ} \mathrm{C}$. The overall optimal parameters create a lattice structure, whose mechanical performance will be compared to the lattice structures fabricated by process parameters in the Taguchi array.
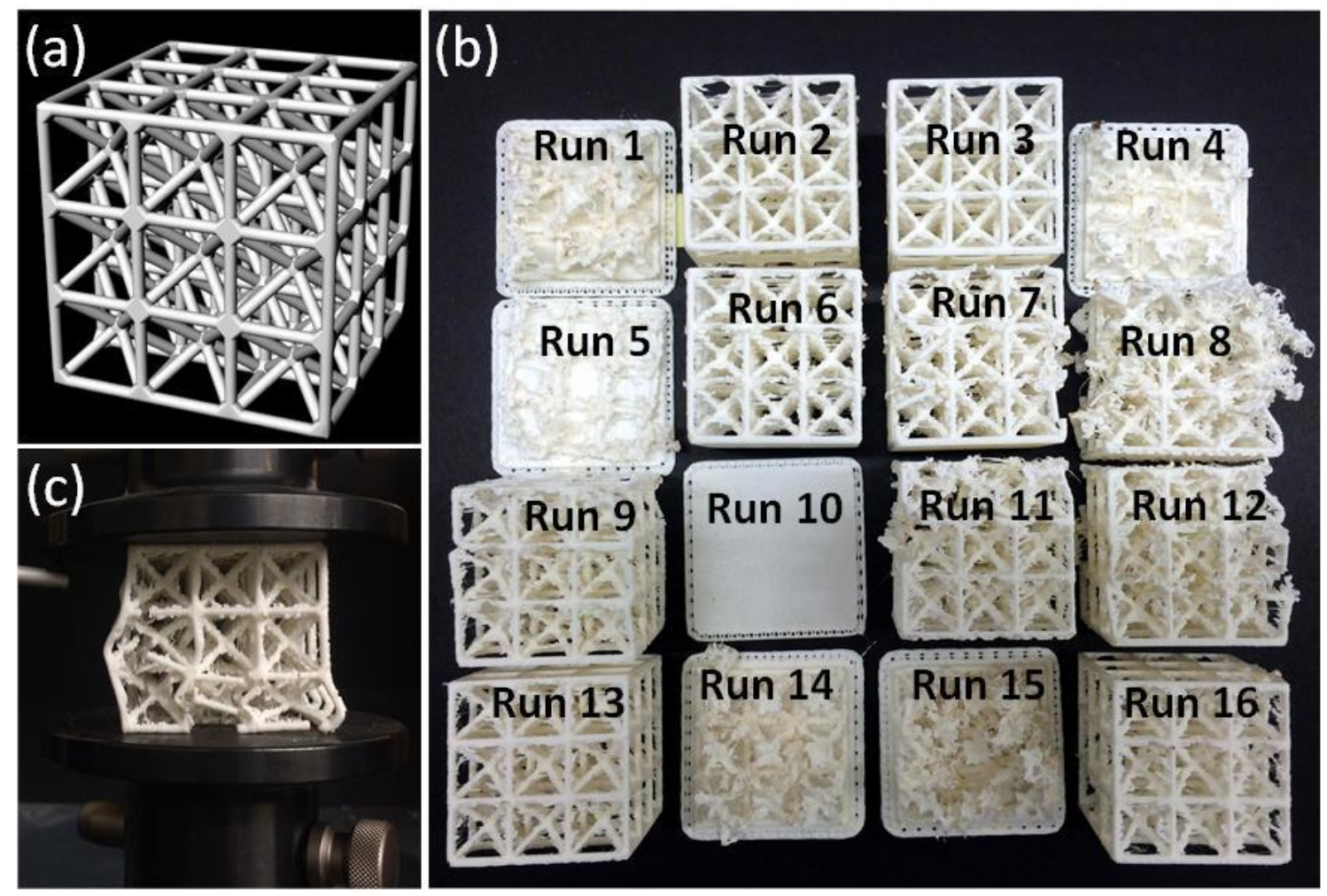

Figure 12 The lattice structure for compression test, (a) the geometrical model, (b) the as built lattice structure by the process parameters in the Taguchi array, (c) the failure of the lattice structure during the compression test.

\subsubsection{Test result and discussion}

The compression test is conducted by a TestResources 313 Universal Test Machine as shown in Figure $12(\mathrm{c})$ at a strain rate of $2 \mathrm{~mm} / \mathrm{min}$. The loading direction is perpendicular to the build direction of the FDM machine coordinate system for two reasons. The first is that the bonding strength between each layer of the lattice structure can be tested in this direction. Because loading along the build direction would compress each layer, which does not expose the weak bonding strength between layers. The second reason is that the horizontal strut deflects during the fabrication process, which is vulnerable to bulking. The compression load perpendicular to the build direction causes bulking on horizontal struts so that manufacturing defects are more clearly seen.

The results of the compression test are shown in Figure 13, with no test results for the failed lattice structures. The effective elastic modulus and the ultimate strength are summarized in Figure 14. It can be concluded that lattice structures fabricated with different process parameters are likely to exhibit different mechanical properties. However, the lattice structure fabricated by the optimized 
process parameters exhibits the highest effective Young's modulus and ultimate strength. Also, Lattice structures from Runs 2, 3 and 13 demonstrate better mechanical performance compared to the other runs. This is because all these runs have a $0.2 \mathrm{~mm}$ layer thickness, the most significant parameter at the optimal level for horizontal struts. Furthermore, Runs 2 and 13 have 50\% fan speed, which is the most significant parameter at optimal level for inclined struts; Run 3 with its $100 \%$ fan speed had lower effective Young's modulus and ultimate strength compared to Runs 2 and 13. In addition, while lattice structures are successfully fabricated in Runs 6, 7,9 and 12, they exhibit worse mechanical performance compared to Runs 2 and 13 because their $0 \%$ fan speed is the worst level for inclined struts. Therefore, the optimal level of process parameters from $\mathrm{S} / \mathrm{N}$ ratio analysis, and most significant process parameters from ANOVA can be used to improve the mechanical performance of as built lattice structure.

Another conclusion is that all the lattice structures fabricated with $0.1 \mathrm{~mm}$ layer thickness fail except in Run 11 where the other process parameters are exact or close to the optimal level. As a result, layer thickness is the most significant parameter in the fabrication of lattice structure by FDM process.

One main failure mechanism in the compression test of lattice structures is fracture between layers of the vertical strut as shown in Figure 12(c). A higher nozzle temperature will strengthen the bond between each layer in the FDM process. The Optimized Run has the same process parameter levels as Run 2, except a nozzle temperature that is $25^{\circ} \mathrm{C}$ higher, which is reflected in a $12 \%$ higher ultimate strength in the Optimized Run compared to Run 2.

Another important failure mechanism in Figure 12(c) is the bulking of the horizontal strut. Some horizontal struts are badly fabricated with large deflection, making them vulnerable to bulking when the compression force is along their orientation. This can be seen by comparing Run 13 and the Optimized Run. While they both have the same layer thickness and fan speed, the Optimized Run's $600 \mathrm{~mm} / \mathrm{min}$ printing speed is the best level for horizontal struts and Run 13 uses $1200 \mathrm{~mm} / \mathrm{min}$. In addition, the nozzle temperature in the Optimized Run is $250^{\circ} \mathrm{C}$ which is closer to the optimal nozzle temperature for horizontal struts. Consequently, the effective Young's modulus and ultimate strength in the Optimized Run are $13 \%$ and $8 \%$ higher respectively than those in Run13. 


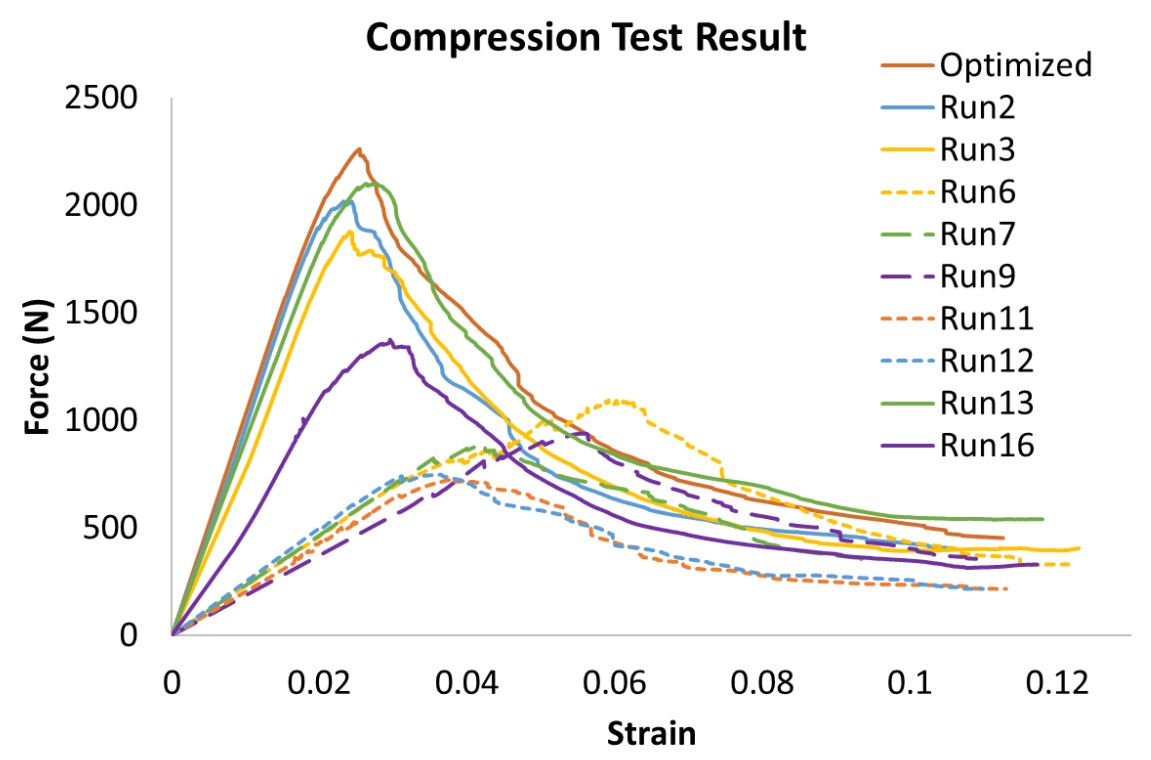

Figure 13 The result of the compression test

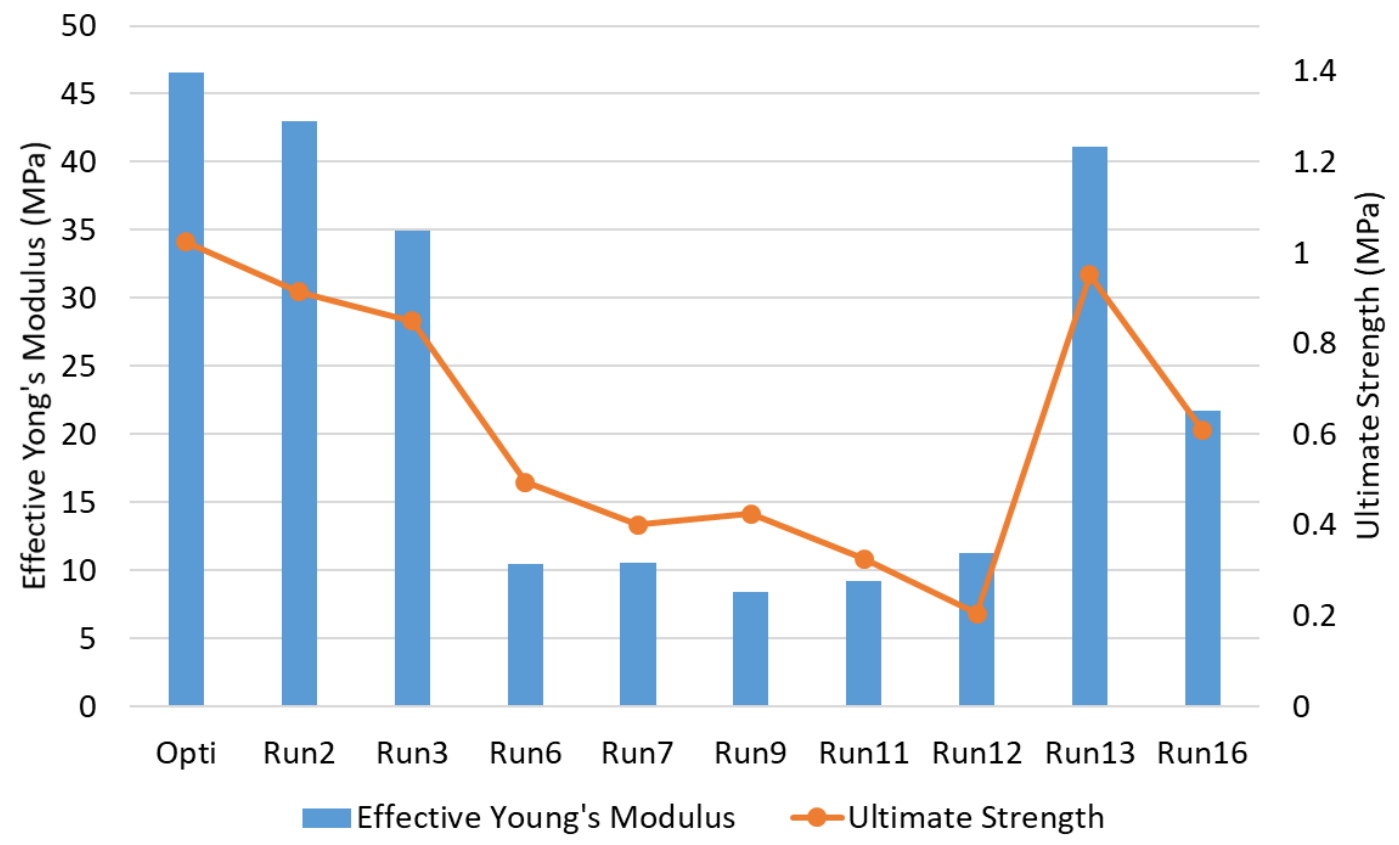

Figure 14 The comparison of the effective young's modulus and ultimate strength of lattice structures fabricated with different process parameters

\section{Conclusions}

In this paper, the process parameters of FDM have been optimized for lattice structures. The concept of the Manufacturable Element is proposed to link the geometrical information of lattice structures and the manufacturing process. The lattice structure is deconstructed into horizontal and inclined struts. Taguchi method is implemented to investigate the optimal process parameters for both types of struts. Failure methods are used to represent the data of failed fabrication. It is shown 
that except for omitted method, the other three failure methods can well represent the missing data. $\mathrm{S} / \mathrm{N}$ ratio analysis and ANOVA has been used to optimize the process parameter. The result shows that the most significant process parameter for inclined struts is fan speed; but for horizontal struts it is layer height. The optimal values for inclined struts are $255^{\circ} \mathrm{C}$ nozzle temperature, $1200 \mathrm{~mm} / \mathrm{min}$ print speed, $50 \%$ fan speed and $0.1 \mathrm{~mm}$ layer height. However, for horizontal struts, the optimum is $245^{\circ} \mathrm{C}$ nozzle temperature, $600 \mathrm{~mm} / \mathrm{min}$ print speed, $0 \%$ fan speed and $0.2 \mathrm{~mm}$ layer height. Furthermore, compression tests have been conducted to investigate the mechanical performance of lattice structures fabricated by different process parameters. Experimental result shows that the proposed optimization method can improve the mechanical performance of lattice structure, even though the optimization is based on the printing quality of lattice struts. All fabrications in this research use constant process parameters. Nevertheless, the $\mathrm{S} / \mathrm{N}$ ratio analysis shows that optimized process parameters are different for different features. Future work will investigate the printing quality of lattice structures fabricated by FDM via dynamic process parameters.

\section{Acknowledgement}

This research work is supported by National Sciences and Engineering Research Council of Canada Discovery Grant RGPIN436055-2013.

\section{Reference}

[1] V. Deshpande, N. Fleck, M. Ashby, Effective properties of the octet-truss lattice material, Journal of the Mechanics and Physics of Solids, 49 (2001) 1747-1769.

[2] J. Lehman, R. Lakes, Stiff lattices with zero thermal expansion and enhanced stiffness via rib cross section optimization, International Journal of Mechanics and Materials in Design, 9 (2013) 213-225.

[3] L. Yang, D. Cormier, H. West, O. Harrysson, K. Knowlson, Non-stochastic Ti-6AI-4V foam structures with negative Poisson's ratio, Materials Science and Engineering A, 558 (2012) 579585.

[4] Q. Zhang, Y. Han, C. Chen, T. Lu, Ultralight X-type lattice sandwich structure (I): Concept, fabrication and experimental characterization, Science in China, Series E: Technological Sciences, 52 (2009) 2147-2154.

[5] Y. Tang, A. Kurtz, Y.F. Zhao, Bidirectional Evolutionary Structural Optimization (BESO) based design method for lattice structure to be fabricated by additive manufacturing, Computer-Aided Design, 69 (2015) 91-101.

[6] D.W. Rosen, Computer-aided design for additive manufacturing of cellular structures, Computer-Aided Design \& Applications, 4 (2007) 585-594.

[7] Z. Ozdemir, E. Hernandez-Nava, A. Tyas, J.A. Warren, S.D. Fay, R. Goodall, I. Todd, H. Askes, Energy absorption in lattice structures in dynamics: Experiments, International Journal of Impact Engineering, 89 (2016) 49-61.

[8] J. Lehman, R.S. Lakes, Stiff, strong, zero thermal expansion lattices via material hierarchy, Composite Structures, 107 (2014) 654-663.

[9] H. Brooks, K. Brigden, Design of conformal cooling layers with self-supporting lattices for additively manufactured tooling, Additive Manufacturing, 11 (2016) 16-22.

[10] S. Arabnejad Khanoki, D. Pasini, Fatigue design of a mechanically biocompatible lattice for a proof-of-concept femoral stem, Journal of the Mechanical Behavior of Biomedical Materials, 22 (2013) 65-83. 
[11] S.H. Masood, J.P. Singh, Y. Morsi, The design and manufacturing of porous scaffolds for tissue engineering using rapid prototyping, International Journal of Advanced Manufacturing Technology, 27 (2005) 415-420.

[12] C.M. Cheah, C.K. Chua, K.F. Leong, S.W. Chua, Development of a Tissue Engineering Scaffold Structure Library for Rapid Prototyping. Part 1: Investigation and Classification, The International Journal of Advanced Manufacturing Technology, 21 (2003) 291-301.

[13] H.N.G. Wadley, Multifunctional periodic cellular metals, Philosophical Transactions of the Royal Society A: Mathematical, Physical and Engineering Sciences, 364 (2006) 31-68.

[14] C.W. Lee, C.K. Chua, C.M. Cheah, L.H. Tan, C. Feng, Rapid investment casting: Direct and indirect approaches via fused deposition modelling, International Journal of Advanced Manufacturing Technology, 23 (2004) 93-101.

[15] C. Yan, L. Hao, A. Hussein, D. Raymont, Evaluations of cellular lattice structures manufactured using selective laser melting, International Journal of Machine Tools and Manufacture, 62 (2012) 32-38.

[16] J. Parthasarathy, B. Starly, S. Raman, A. Christensen, Mechanical evaluation of porous titanium (Ti6Al4V) structures with electron beam melting (EBM), Journal of the Mechanical Behavior of Biomedical Materials, 3 (2010) 249-259.

[17] M.R. Karamooz Ravari, M. Kadkhodaei, M. Badrossamay, R. Rezaei, Numerical investigation on mechanical properties of cellular lattice structures fabricated by fused deposition modeling, International Journal of Mechanical Sciences, 88 (2014) 154-161.

[18] E. Atzeni, A. Salmi, Study on unsupported overhangs of AlSi10Mg parts processed by Direct Metal Laser Sintering (DMLS), Journal of Manufacturing Processes, 20 (2015) 500-506.

[19] J.C. Fox, S.P. Moylan, B.M. Lane, Effect of Process Parameters on the Surface Roughness of Overhanging Structures in Laser Powder Bed Fusion Additive Manufacturing, in: Procedia CIRP, 2016, pp. 131-134.

[20] Y. Pupo, K.P. Monroy, J. Ciurana, Influence of process parameters on surface quality of CoCrMo produced by selective laser melting, International Journal of Advanced Manufacturing Technology, 80 (2015) 985-995.

[21] C. Kamath, B. El-Dasher, G.F. Gallegos, W.E. King, A. Sisto, Density of additivelymanufactured, 316L SS parts using laser powder-bed fusion at powers up to $400 \mathrm{~W}$, International Journal of Advanced Manufacturing Technology, 74 (2014) 65-78.

[22] T.M. Wang, J.T. Xi, Y. Jin, A model research for prototype warp deformation in the FDM process, International Journal of Advanced Manufacturing Technology, 33 (2007) 1087-1096. [23] R.K. Roy, A primer on the Taguchi method, Society of Manufacturing Engineers, 2010. [24] R. Anitha, S. Arunachalam, P. Radhakrishnan, Critical parameters influencing the quality of prototypes in fused deposition modelling, Journal of Materials Processing Technology, 118 (2001) 385-388.

[25] B.H. Lee, J. Abdullah, Z.A. Khan, Optimization of rapid prototyping parameters for production of flexible ABS object, Journal of Materials Processing Technology, 169 (2005) 54-61. [26] Z.A.K.a.S.Y.K. J. Laeng, Optimizing Flexible Behaviour of Bow Prototype Using Taguchi Approach, (2006).

[27] W. Che Chung, L. Ta - Wei, H. Shr - Shiung, Optimizing the rapid prototyping process by integrating the Taguchi method with the Gray relational analysis, Rapid Prototyping Journal, 13 (2007) 304-315.

[28] A.K. Sood, R.K. Ohdar, S.S. Mahapatra, Improving dimensional accuracy of Fused Deposition Modelling processed part using grey Taguchi method, Materials \& Design, 30 (2009) 4243-4252. 
[29] X. Liu, M. Zhang, S. Li, L. Si, J. Peng, Y. Hu, Mechanical property parametric appraisal of fused deposition modeling parts based on the gray Taguchi method, International Journal of Advanced Manufacturing Technology, (2016) 1-11.

[30] Y. Tang, G. Dong, Q. Zhou, Y.F. Zhao, Lattice Structure Design and Optimization With Additive Manufacturing Constraints, IEEE Transactions on Automation Science and Engineering, PP (2017) 1-17.

[31] D.W. Rosen, Computer-Aided Design for Additive Manufacturing of Cellular Structures, Computer-Aided Design and Applications, 4 (2007) 585-594.

[32] G. Taguchi, S. Chowdhury, Y. Wu, Taguchi's Quality Engineering Handbook, 2007.

[33] R. Christensen, Analysis of variance, design, and regression: applied statistical methods, CRC Press, 1996.

\section{Appendix}

Appendix Table 1. S/N values for inclines (decibels)

\begin{tabular}{|l|l|l|l|l|l|}
\hline \multirow{2}{*}{ Process and Design Parameters } & \multicolumn{4}{l}{ Failure method } \\
\cline { 2 - 6 } & 225 & 7.802 & 6.979 & 6.880 & 6.301 \\
\hline \multirow{5}{*}{ Temperature $\left({ }^{\circ} \mathrm{C}\right)$} & 235 & 8.384 & 8.384 & 8.384 & 8.384 \\
\cline { 2 - 6 } & 245 & 8.843 & 8.843 & 8.843 & 8.843 \\
\cline { 2 - 6 } & 255 & 9.744 & 9.744 & 9.744 & 9.744 \\
\hline \multirow{5}{*}{ Print Speed (mm/s) } & 600 & 8.855 & 8.810 & 8.797 & 8.595 \\
\cline { 2 - 6 } & 1200 & 9.339 & 9.339 & 9.339 & 9.339 \\
\cline { 2 - 6 } & 1800 & 8.833 & 8.612 & 8.524 & 8.398 \\
\cline { 2 - 6 } & 2400 & 7.765 & 7.188 & 7.190 & 6.939 \\
\hline \multirow{5}{*}{ Fan Speed $(\%)$} & 0 & 5.939 & 5.667 & 5.661 & 5.435 \\
\cline { 2 - 6 } & 50 & 11.720 & 11.720 & 11.720 & 11.720 \\
\cline { 2 - 6 } & 100 & 11.165 & 10.895 & 10.807 & 10.682 \\
\hline \multirow{3}{*}{ Layer Height (mm) } & 0.1 & 8.841 & 8.508 & 8.502 & 8.275 \\
\cline { 2 - 6 } & 0.2 & 8.575 & 8.467 & 8.423 & 8.360 \\
\hline \multirow{5}{*}{ Diameter (mm) } & 15 & -3.310 & -3.482 & -3.334 & -3.545 \\
\cline { 2 - 6 } & 30 & 3.707 & 3.459 & 3.212 & 2.844 \\
\cline { 2 - 6 } & 60 & 14.068 & 14.068 & 14.068 & 14.068 \\
\cline { 2 - 6 } & 90 & 19.904 & 19.904 & 19.904 & 19.904 \\
\hline \multirow{5}{*}{ Length (mm) } & 2 & 7.682 & 7.070 & 6.996 & 6.562 \\
\cline { 2 - 6 } & 4 & 8.564 & 8.564 & 8.564 & 8.564 \\
\cline { 2 - 6 } & 6 & 9.828 & 9.828 & 9.828 & 9.828 \\
\hline
\end{tabular}

Appendix Table 2. S/N values for horizontal struts (decibels)

\begin{tabular}{|l|l|l|l|l|l|}
\hline \multirow{2}{*}{ Process and Design Parameters } & \multicolumn{5}{l}{ Failure method } \\
\cline { 3 - 7 } & 225 & 2.566 & -0.727 & -2.309 & -4.817 \\
\hline \multirow{4}{*}{ Temperature $\left({ }^{\circ} \mathrm{C}\right)$} & 235 & 2.809 & 1.436 & 0.787 & 0.192 \\
\cline { 2 - 7 } & 245 & 3.591 & 2.042 & 1.883 & 0.777 \\
\cline { 2 - 7 } & 255 & 2.975 & 1.280 & 0.934 & -0.550 \\
\hline \multirow{5}{*}{ Print Speed $(\mathrm{mm} / \mathrm{s})$} & 600 & 2.426 & 2.420 & 2.402 & 2.402 \\
\cline { 2 - 7 } & 1200 & 3.477 & 0.704 & 0.480 & -1.364 \\
\cline { 2 - 7 } & 1800 & 3.133 & 0.651 & 0.307 & -1.489 \\
\cline { 2 - 6 } & 2400 & 3.192 & 0.257 & -1.894 & -3.948 \\
\hline Fan Speed $(\%)$ & 0 & 3.175 & 1.068 & 0.076 & -1.442 \\
\hline
\end{tabular}




\begin{tabular}{|l|l|l|l|l|l|}
\hline & 50 & 3.451 & 0.998 & 0.382 & -0.967 \\
\cline { 2 - 6 } & 100 & 2.331 & 0.897 & 0.760 & -0.548 \\
\hline \multirow{3}{*}{ Layer Height (mm) } & 0.1 & 0.788 & -1.194 & -2.468 & -4.421 \\
\cline { 2 - 6 } & 0.2 & 4.732 & 3.210 & 3.116 & 2.221 \\
\hline \multirow{3}{*}{ Diameter (mm) } & 2 & -0.184 & -3.355 & -3.581 & -6.860 \\
\cline { 2 - 6 } & 4 & 2.545 & 1.651 & 0.783 & 0.292 \\
\cline { 2 - 6 } & 6 & 5.520 & 4.727 & 3.770 & 3.270 \\
\hline \multirow{3}{*}{ Length (mm) } & 10 & 10.020 & 7.189 & 5.808 & 4.554 \\
\cline { 2 - 6 } & 30 & 2.682 & 0.687 & -0.045 & -1.701 \\
\cline { 2 - 6 } & 50 & -3.505 & -4.853 & -4.792 & -6.152 \\
\hline
\end{tabular}

Appendix Table 3. ANOVA for means for inclines

\begin{tabular}{|c|c|c|c|c|c|c|}
\hline \multicolumn{6}{|c|}{ Failure method: max deviation } & \multirow[b]{2}{*}{ Rank } \\
\hline Factor & DOF & SS & MS & F ratio & $\mathrm{P}$ & \\
\hline Temperature & 3 & 6.151 & 2.050 & 3.462 & 0.0872 & 2 \\
\hline Speed & 3 & 1.953 & 0.651 & 1.099 & 0.0277 & 3 \\
\hline Fan & 2 & 39.753 & 19.876 & 33.563 & 0.8456 & 1 \\
\hline Layer Height & 1 & 0.3 & 0.335 & 0.566 & 0.0143 & 4 \\
\hline Error & 566 & 335.19 & 0.592 & & 0.0252 & \\
\hline Total & 575 & 383.39 & & & & \\
\hline \multicolumn{7}{|c|}{ Failure method: $2 *$ strut diameter } \\
\hline Fact & DOF & SS & MS & F ratio & $\mathrm{P}$ & \\
\hline Ter & 3 & 17.094 & 5.698 & 6.831 & 0.1739 & 2 \\
\hline Speed & 3 & 4 & 1.394 & 1.671 & 0.0425 & 3 \\
\hline Fan & 2 & 48.713 & 24.357 & 29.201 & 0.7433 & 1 \\
\hline Layer Height & 1 & 0.487 & 0.487 & 0.584 & 0.0149 & 4 \\
\hline Erro & 566 & 472.11 & 0.834 & & 0.0255 & \\
\hline Total & 575 & 542.58 & & & & \\
\hline \multicolumn{7}{|c|}{ Failure method: $1 *$ strut diameter } \\
\hline Factor & DOF & SS & MS & F ratio & $\mathrm{P}$ & \\
\hline Ten & 3 & & 2.062 & 3.741 & 79 & 2 \\
\hline Speec & 3 & & 0.449 & 0.814 & 0.0213 & 3 \\
\hline Fan & 2 & 35.425 & 17.712 & 32.130 & 0.8412 & 1 \\
\hline Layer Height & 1 & 0.282 & 0.282 & 0.512 & 0.0134 & 4 \\
\hline Error & 566 & 312.02 & 0.551 & & 0.0262 & \\
\hline Total & 575 & 355.26 & & & & \\
\hline
\end{tabular}

Appendix Table 4. ANOVA for means for horizontal struts 


\begin{tabular}{|lrrrrr|r|}
\hline Failure method: max deviation & & & & \\
\hline Factor & DOF & SS & MS & F ratio & P & Rank \\
Temperature & 3 & 10.803 & 3.601 & 5.574 & 0.0993 & 2 \\
Speed & 3 & 3.463 & 1.154 & 1.787 & 0.0318 & 4 \\
Fan & 2 & 2.446 & 1.223 & 1.893 & 0.0337 & 3 \\
Layer Height & 1 & 29.634 & 29.634 & 45.872 & 0.8173 & 1 \\
Error & 422 & 272.62 & 0.646 & & 0.0178 & \\
Total & 431 & 318.97 & & & & \\
\hline Failure method: $\mathbf{2}^{*}$ strut diameter & & & \\
\hline Factor & DOF & SS & MS & F ratio & P & \\
Temperature & 3 & 272.087 & 90.696 & 7.747 & 0.2030 & 2 \\
Speed & 3 & 233.641 & 77.880 & 6.652 & 0.1743 & 3 \\
Fan & 2 & 12.466 & 6.233 & 0.532 & 0.0140 & 4 \\
Layer Height & 1 & 260.234 & 260.234 & 22.229 & 0.5825 & 1 \\
Error & 134 & 1568.73 & 11.707 & & 0.0262 & \\
Total & 143 & 2347.15 & & & & \\
\hline Failure method: 1* strut diameter & & & & \\
\hline Factor & DOF & SS & MS & F ratio & P & Rank \\
Temperature & 3 & 53.737 & 17.912 & 17.399 & 0.1520 & 2 \\
Speed & 3 & 49.300 & 16.434 & 15.962 & 0.1394 & 3 \\
Fan & 2 & 1.072 & 0.536 & 0.520 & 0.0045 & 4 \\
Layer Height & 1 & 81.942 & 81.942 & 79.593 & 0.6953 & 1 \\
Error & 422 & 434.46 & 1.030 & & 0.0087 & \\
Total & 431 & 620.51 & & & & \\
\hline
\end{tabular}

